\title{
Interlayer Exchange Coupling in Semiconductor EuS-PbS Ferromagnetic Wedge Multilayers
}

\author{
L. Kowalczyk ${ }^{a}, \mathrm{~S} . \mathrm{WroteK}^{a}, \mathrm{P} \cdot \mathrm{Dziawa}^{a}, \mathrm{~V} \cdot \mathrm{OSInNiY}^{a}$,

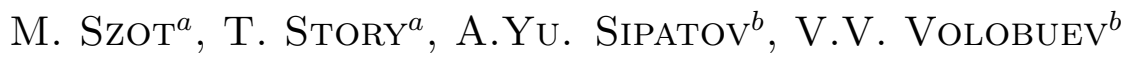 \\ AND A.G. FEDOROV ${ }^{c}$ \\ ${ }^{a}$ Institute of Physics, Polish Academy of Sciences \\ al. Lotników 32/46, 02-668 Warsaw, Poland \\ ${ }^{b}$ National Technical University KPI \\ 21 Frunze Street, 61002 Kharkov, Ukraine \\ ${ }^{c}$ Institute for Scintillation Materials NASU \\ Lenin Ave. 60, 61001 Kharkov, Ukraine
}

\begin{abstract}
Antiferromagnetic interlayer coupling between ferromagnetic layers of EuS via nonmagnetic PbS spacer layer was experimentally studied in EuS$\mathrm{PbS}$ wedge multilayers grown on $\mathrm{KCl}$ (001) substrates with EuS thickness of $6 \mathrm{~nm}$ and $\mathrm{PbS}$ thickness varying in the wedges in the range $0.3-6 \mathrm{~nm}$ (i.e. $n=1-20$ monolayers). Measurements of magnetic hysteresis loops of EuS-PbS multilayers performed in the temperature range $5-30 \mathrm{~K}$ by superconducting (SQUID) and magneto-optical magnetometers revealed a rapid increase in saturation magnetic field in multilayers with $\mathrm{PbS}$ spacer thinner than about $1.5 \mathrm{~nm}$. It shows a monotonic increase in interlayer coupling strength with a decreasing $\mathrm{PbS}$ spacer thickness, in qualitative agreement with $1 / 2^{n}$ dependence predicted theoretically for semiconductor magnetic superlattices.
\end{abstract}

PACS numbers: 75.20.Ck, 75.30.Et

\section{Introduction}

In EuS-PbS semiconductor heterostructures ferromagnetic layers of EuS are separated by crystal lattice-matched nonmagnetic (diamagnetic) layers of $\mathrm{PbS}$. Both materials crystallize in the rock-salt crystal structure with lattice parameters $0.597 \mathrm{~nm}$ and $0.594 \mathrm{~nm}$ for $\mathrm{EuS}$ and $\mathrm{PbS}$, respectively, and are known to form good quality epitaxial heterostructures [1]. PbS belongs to the well known IV-VI family of semiconductor compounds and is characterized by narrow energy 
gap of $0.3 \mathrm{eV}$ (at low temperatures) and $n$-type conductivity (typical electron concentration of about $10^{18} \mathrm{~cm}^{-3}$ ). EuS is a magnetic semiconductor constituting a unique example of a model Heisenberg (insulating) ferromagnet. As EuS has much wider energy gap (1.65 eV) than PbS, in EuS-PbS multilayers EuS layers form energy barriers for PbS quantum wells [2-4]. Ferromagnetic transition temperature in bulk EuS crystals equals $16.5 \mathrm{~K}$ whereas in EuS epitaxial layers grown on $\mathrm{KCl}$ substrates the Curie temperature increases to $17.5 \mathrm{~K}$ due to thermal strain brought about by the difference of thermal expansion coefficients of the layer and the substrate $[1,2]$. In EuS layers thinner than about $3 \mathrm{~nm}$ the size effect in magnetic properties is also observed $[2,5]$ with a Curie temperature reduced in ultrathin $(0.6 \mathrm{~nm})$ layers down to about $2 / 3$ of its value in $0.1 \mu \mathrm{m}$ thick layers. It was found experimentally that in $\mathrm{EuS}-\mathrm{PbS}$ semiconductor multilayers with ultrathin (about $1 \mathrm{~nm}$ ) PbS layer, the ferromagnetic layers of EuS are coupled antiferromagnetically via the nonmagnetic $\mathrm{PbS}$ spacer. This effect was first observed in neutron reflectivity [6] and magnetization [7-10] studies of EuS-PbS superlattices grown along the [001] direction on $\mathrm{KCl}$ monocrystalline substrates. Very similar effects were later found experimentally and analyzed quantitatively in magnetic hysteresis studies of EuS-PbS-EuS trilayers grown both on $\mathrm{KCl}$ (001) and $\mathrm{PbS}$ (001) substrates [7, 8]. Antiferromagnetic interlayer exchange coupling was also experimentally observed in closely related EuS-YbSe superlattices in which nonmagnetic YbSe spacer material has a band gap similar to EuS layer $[9,10]$. It is interesting to note that the antiferromagnetic interlayer coupling was not found experimentally in neither EuS-PbS superlattices nor trilayers grown on $\mathrm{BaF}_{2}$ substrates with [111] growth direction. A variety of theoretical models was proposed to explain antiferromagnetic exchange coupling in magnetic semiconductor superlattices [11]. The standard Ruderman-Kittel-Kasuya-Yoshida (RKKY) mechanism well known in metallic multilayers proceeds via free carriers and to be efficient requires at least $10^{20} \mathrm{~cm}^{-3}$ of conducting electrons or holes. This mechanism was analyzed in Ref. [12] taking into account important modifications related to low carrier concentration regime and two-dimensional character of free electron gas in these semiconductor materials. As this modified RKKY mechanism predicts in EuS-PbS system mostly ferromagnetic coupling and is efficient only for high electron concentration, it is not likely to explain the experimental findings. Particularly relevant for EuS-PbS multilayers is the model of Blinowski and Kacman $(\mathrm{B}-\mathrm{K})$ in which the interlayer coupling is related to the spin polarization of valence band electrons (with no free carriers present) [11]. The key predictions of this model concerning the interlayer coupling are: its antiferromagnetic sign, a rapid exponential decay with increasing spacer thickness, an important role of band gap value of nonmagnetic spacer material, and practically no dependence on magnetic layer thickness. In realistic models of multilayer structures the effects of dipolar interactions brought about e.g. by interface roughness or domain walls need also to be analyzed. 
In the experimental verification of various physical mechanisms of interlayer coupling in EuS-PbS multilayers the key experimental problem remains the determination of the dependence of the coupling mechanism on the spacer thickness. In this work, we study experimentally the effect of antiferromagnetic interlayer coupling on magnetic hysteresis loops of EuS-PbS superlattices grown on $\mathrm{KCl}$ (001) substrate in the form of wedges with $\mathrm{PbS}$ spacer thickness varying in a continuous way in the range $0.3-6 \mathrm{~nm}$ (i.e. 1-20 monolayers (ML)). The magnetization of these structures is studied employing both superconducting quantum interference device (SQUID) and magneto-optical Kerr effect (MOKE) magnetometry techniques in order to gain new information on the spacer thickness dependence of the coupling permitting the verification of various interlayer exchange coupling mechanisms.

\section{Experimental}

$\operatorname{EuS}(6 \mathrm{~nm})-\mathrm{PbS}(d)$ superlattice wedge structures were grown employing high vacuum deposition facility $\left(10^{-7}\right.$ Torr $)$ at Kharkov Technical University equipped with electron gun for EuS deposition and resistively heated tungsten boat for $\mathrm{PbS}$ deposition. To prepare EuS-PbS wedge superlattice structures with $\mathrm{PbS}$ spacer thickness covering the most important range 1-20 ML (0.3-6 nm), 4 wedge structures were grown covering the $\mathrm{PbS}$ thickness range from 0.3 to $0.7 \mathrm{~nm}$, $0.6-1.5 \mathrm{~nm}, 1.5-3 \mathrm{~nm}$, and 3-6 nm. The layers were epitaxially deposited on the freshly cleaved $\mathrm{KCl}$ (001) monocrystalline substrates with 10 times repeated basic EuS-PbS bilayer. All the multilayers contain $30 \mathrm{~nm}$ thick $\mathrm{PbS}$ buffer layer to accommodate $\mathrm{KCl}-\mathrm{PbS}$ lattice parameter misfit as well as thin $(1-6 \mathrm{~nm}) \mathrm{PbS}$ capping layer for preventing the structure from oxidation. The varying $\mathrm{PbS}$ spacer thickness was achieved by positioning the $\mathrm{KCl}$ substrate close to the $\mathrm{PbS}$ vapor source at a certain angle with respect to its axis creating a proper change of $\mathrm{PbS}$ flux over the $3 \mathrm{~cm}$ long $\mathrm{KCl}$ substrate. The electron gun with EuS was located at longer distance providing a uniform EuS flux over the entire substrate. An independent verification of the thickness of EuS layer was obtained from saturation magnetic moment per unit area of the structure. The results of careful X-ray diffraction analysis of structural parameters of the EuS-PbS wedge multilayers are presented in Ref. [13]. The diffraction spectra reveal in the EuS-PbS wedge superlattices satellite peaks up to the 4th order indicating the high crystal quality and excellent agreement of EuS-PbS superlattice period with technological data from in situ quartz layer thickness monitor.

The magnetization measurements of small (about $0.1 \mathrm{~cm}^{2}$ ) pieces of the wedge structure were carried out by SQUID magnetometer in the temperature range $2-25 \mathrm{~K}$ and magnetic field range up to $250 \mathrm{Oe}$, i.e. sufficient to fully magnetically saturate the EuS layers. In agreement with our previous experimental studies of EuS-PbS multilayers grown on $\mathrm{KCl}$ substrates [2, 6-8], the superlattices undergo a ferromagnetic transition at about $17 \mathrm{~K}$ as shown in temperature 
dependence of magnetic hysteresis loops (Fig. 1). The measurements of hysteresis loops were carried out applying an external magnetic field in the (001) plane of the layer along [100] crystal axis. Magnetic hysteresis loops of $\operatorname{EuS}(6 \mathrm{~nm})-\mathrm{PbS}(3 \mathrm{~nm})$ and $\operatorname{EuS}(6 \mathrm{~nm})-\operatorname{PbS}(0.7 \mathrm{~nm})$ superlattices at $T=5 \mathrm{~K}$ are presented in Fig. 2.

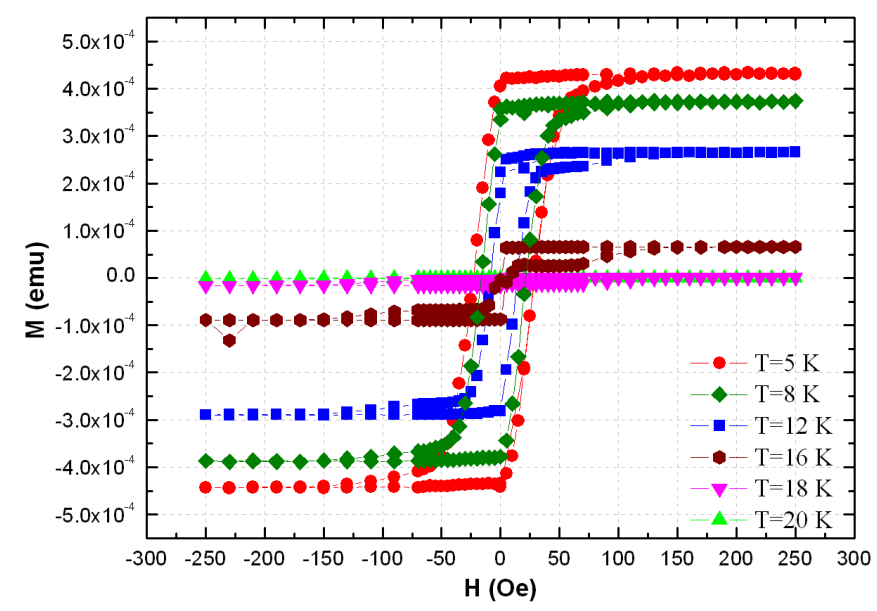

Fig. 1. Ferromagnetic transition in EuS-PbS multilayer with $1.5 \mathrm{~nm}$ thick $\mathrm{PbS}$ spacer as observed in temperature dependence of magnetic hysteresis loop.

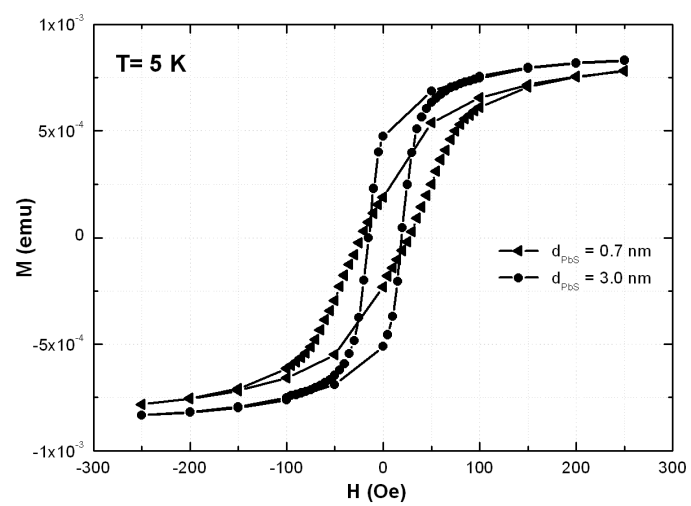

Fig. 2. Magnetic hysteresis loops of $\operatorname{EuS}(6 \mathrm{~nm})-\operatorname{PbS}(3 \mathrm{~nm})$ and $\operatorname{EuS}(6 \mathrm{~nm})-$ $\mathrm{PbS}(0.7 \mathrm{~nm})$ multilayers showing uncoupled and antiferromagnetically coupled behavior, respectively.

In the multilayers with $\mathrm{PbS}$ spacer thinner than about $1.5 \mathrm{~nm}$ the magnetization loops of wedge superlattices clearly show a strong decrease in magnetic remanence and an increase in saturation field for structures with the $\mathrm{PbS}$ spacer thickness decreasing below $1.5 \mathrm{~nm}$ (see Fig. 3). The coercive field of the layers shows only small increase in the same range of spacer thicknesses [13]. 


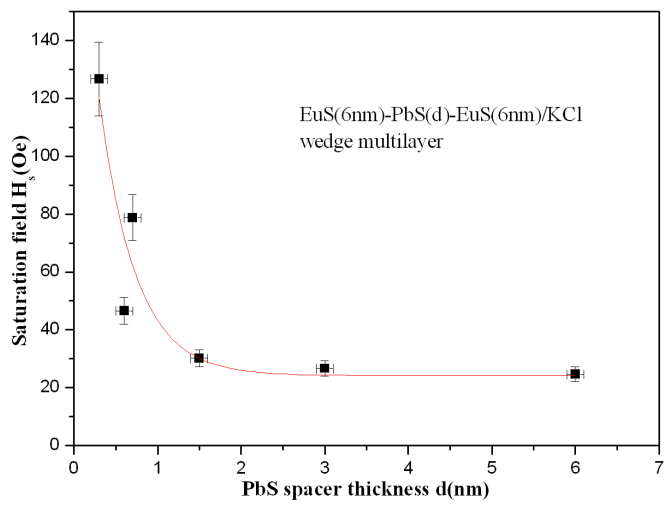

Fig. 3. Saturation magnetic field at $T=5 \mathrm{~K}$ in $\operatorname{EuS}(6 \mathrm{~nm})-\mathrm{PbS}(d)$ multilayers with varying $\mathrm{PbS}$ spacer thickness $d$. The solid line shows $1 / 2^{n}(n=d / 0.3 \mathrm{~nm}$ is the number of monolayers) contribution to the interface exchange interaction predicted in Ref. [11] and a constant background.

For the experimental analysis of the magnetic hysteresis loops along the wedge structure MOKE magnetometry was applied in the temperature range $T=$ 4-30 K and magnetic fields up to 300 Oe using He-Ne laser. The MOKE analysis of the $0.3-0.7 \mathrm{~nm}$ and $0.6-1.5 \mathrm{~nm}$ wedges covering (due to optical limitations of the experimental setup) only about $1 / 2$ of the total wedge length showed only a rather weak continuous evolution of the magnetization loop parameters.

\section{Discussion}

Qualitatively, the magnetization loops of EuS-PbS wedge superlattices are similar to the case of previously studied antiferromagnetically coupled EuS-PbS superlattices and trilayers grown on $\mathrm{KCl}$ (001) substrates [2-6]. However, a characteristic low field linear "plateau" with a well defined external magnetic switching field observed in trilayer structures was not clearly observed in the wedge structures and could only be determined for the EuS-PbS structure with the thinnest $\mathrm{PbS}$ spacer of $0.3 \mathrm{~nm}$. This is related to the use in this work of superlattices instead of simpler trilayer structures. Superlattices are of key importance for reliable X-ray diffraction verification of the wedge structure parameters but also obscure quantitative analysis of magnetic hysteresis loops due to inevitable variation of switching field in 10 times repeated basic bilayer structure. Therefore, the analysis of exchange coupling based on magnetic hysteresis loops measurements of wedge structures can be done by studying the magnetic saturation field $H_{\mathrm{s}}$ which is directly proportional to the interlayer exchange coupling: $I_{\mathrm{IEC}}=M_{\mathrm{s}} H_{\mathrm{s}} t / 4$. Here $M_{\mathrm{S}}$ is the magnetization at saturation, $t$ is the thickness of ferromagnetic EuS layer, and factor 1/4 (instead of usual 1/2 used in trilayers) accounts for the coupling of each EuS layer in superlattice to two neighboring magnetic layers. The experimentally found dependence of saturation field on the thickness of PbS spacer 
is presented in Fig. 3. After subtracting the constant background contribution (see discussion below) one can calculate the coupling energy density. For example, for the wedge superlattice structure with $d=0.3 \mathrm{~nm}$ one finds $I_{\mathrm{IEC}}=0.018 \mathrm{erg} / \mathrm{cm}^{2}$. As the experimentally measured hysteresis loops show in EuS considerable rounding of $M(H)$ dependence close to saturation the magnetic saturation field was determined as the field at which full saturation is obtained following the initial slope of low field $M(H)$ dependence. A good estimate of saturation magnetic moment is obtained as magnetization at $H=250$ Oe.

The line in Fig. 3 that describes well the experimental dependence of $H_{\mathrm{s}}(d)$ was obtained taking into account the $1 / 2^{n}$ contribution of B-K model and a constant term which describes the finite slope of $M(H)$ dependence observed in single EuS layers and EuS-PbS multilayers with thick spacer due to the effect of magnetic anisotropy and strain in the layer. An additional, weakly dependent on spacer thickness, contribution is also expected due to dipolar interactions [11]. The key result of this work presented in Fig. 3 agrees qualitatively with the B-K model. This conclusion is also supported by MOKE magnetometry analysis of magnetization loops along the wedge multilayer which indicated only smooth, monotonic change of hysteresis loop parameters revealing no evidence for any sign or strength oscillations of the coupling for varying spacer thickness, expected e.g. in modified RKKY model.

\section{Conclusions}

Antiferromagnetic interlayer exchange coupling was experimentally observed in $\mathrm{EuS}-\mathrm{PbS}-\mathrm{EuS} / \mathrm{KCl}$ (001) ferromagnetic semiconductor wedge multilayers with ultrathin nonmagnetic PbS spacer (below $1.5 \mathrm{~nm}$ ). SQUID magnetometry analysis of saturation field of magnetization loops of EuS-PbS multilayers provided a quantitative estimate for the coupling strength. An experimentally found sharp decrease in saturation field for increasing $\mathrm{PbS}$ spacer thickness can be quantitatively understood in terms of the $\mathrm{B}-\mathrm{K}$ model with a background contribution of dipolar interactions and crystalline anisotropy effects.

\section{Acknowledgments}

The work of V.V. Volobuev was supported by Ukrainian President grant for young scientists research GP/F11/0010.

\section{References}

[1] A. Stachow-Wójcik, T. Story, W. Dobrowolski, M. Arciszewska, R.R. Gałązka, H.J.M. Swagten, W.J.M. de Jonge, A. Twardowski, A.Yu. Sipatov, Phys. Rev. B 60, 15220 (1999).

[2] T. Story, Phys. Status Solidi B 236, 310 (2003).

[3] I.V. Kolesnikov, V.A. Litvinov, A.Yu. Sipatov, A.I. Fedorenko, A.E. Yunovich, Sov. Phys.-JETP 67, 1431 (1988). 
[4] L. Kowalczyk, J. Sadowski, R.R. Gałązka, A. Stachow-Wójcik, A.Yu. Sipatov, V.A. Smirnov, Acta Phys. Pol. A 94, 357 (1998).

[5] R. Świrkowicz, T. Story, J. Phys., Condens. Matter 12, 8511 (2000).

[6] H. Kȩpa, J. Kutner-Pielaszek, J. Blinowski, A. Twardowski, C.F. Majkrzak, T. Story, P. Kacman, R.R. Gałązka, K. Ha, H.J.M. Swagten, W.J.M. de Jonge, A.Yu. Sipatov, V.V. Volobuev, T.M. Giebultowicz, Europhys. Lett. 56, 54 (2001).

[7] M. Chernyshova, L. Kowalczyk, A. Szczerbakow, T. Story, C.J.P. Smits, H.J.M. Swagten, C.H.W. Swuste, J.K. Ha, W.J.M. de Jonge, A.Yu. Sipatov, V.V. Volobuev, J. Supercond. 16, 213 (2003).

[8] C.J.P. Smits, A.T. Filip, H.J.M. Swagten, B. Koopmans, W.J.M. de Jonge, M. Chernyshova, L. Kowalczyk, K. Grasza, A. Szczerbakow, T. Story, W. Palosz, A.Yu. Sipatov, Phys. Rev. B 69, 224410 (2004).

[9] P. Sankowski, H. Kępa, P. Kacman, A.Yu. Sipatov, C.F. Majkrzak, T.M. Giebultowicz, Acta Phys. Pol. A 105, 607 (2004).

[10] H. Kȩpa, C.F. Majkrzak, P. Sankowski, P. Kacman, T.M. Giebultowicz, J. Alloys Comp. 401, 238 (2005).

[11] J. Blinowski, P. Kacman, Phys. Rev. B 64, 045302 (2001).

[12] V.V. Zorchenko, A.Yu. Sipatov, V.V. Volobuev, Low Temp. Phys. 29, 1209 (2003).

[13] L. Kowalczyk, V. Osinniy, M. Chernyshova, P. Dziawa, A. Boratyński, T. Story, C.J.P. Smits, H.J.M. Swagten, A.Yu. Sipatov, V.V. Volobuev, J. Alloys Comp., in press. 